\title{
Actinomycosis in a Mucosal Lesion on a Created Perioral Dimple: A Case Report
}

\author{
Ui Geon Kim, Chung Hun Kim, \\ Kyung Suk Kwon, Euna Hwang \\ Department of Plastic and Reconstructive \\ Surgery, Bundang CHA Medical Center, \\ CHA University School of Medicine, \\ Seongnam, Korea
}

No potential conflict of interest relevant to this article was reported.
Facial dimple creation is a simple surgical procedure. Nonetheless, several complications can occur. Actinomycosis is a rare chronic granulomatous infection caused by Actinomyces species. Some conditions that can cause actinomycosis are trauma, oral surgery, and poor dental hygiene. We report a case of actinomycosis that developed on a created facial dimple. A 51-year-old woman presented with a palpable mass on her left cheek that was approximately $1 \mathrm{~cm}$ in size. She had undergone facial dimple-creating surgery on both perioral areas at a local clinic 12 years previously. She had not experienced any problems until she was diagnosed with rheumatoid arthritis and diabetes mellitus about 2 years previously, for which she took leflunomide and methotrexate. The mass was completely excised through an intraoral approach. The specimen was grossly described as a gray-yellow cystic mass containing non-absorbable suture material. The filamentous nature of the Actinomyces organisms was observed in dark-stained foci on a histologic examination, confirming the diagnosis of actinomycosis. Indwelling non-absorbable suture materials may increase the risk for opportunistic infections, such as actinomycosis, in immunocompromised patients. Therefore, plastic surgeons should be aware of a patient's general hygiene, immune condition, and medical history when using these materials.

Keywords Actinomycosis, Immunosuppressive agents, Infection

\section{INTRODUCTION}

Facial dimple creation surgery is a simple procedure that is performed under local anesthesia. Although it is simple to make a facial dimple, several complications can occur, including hematoma, infection, loss of the dimple, hyperpigmentation, and a granulomatous reaction [1].

Actinomycosis is a rare chronic infectious disease caused by $A c$ -

Received: Jul 12, 2017 Revised: Aug 1, 2017 Accepted: Aug 3, 2017 Correspondence: Euna Hwang Department of Plastic and Reconstructive Surgery, Bundang CHA Medical Center, CHA University School of Medicine, 59 Yatap-ro, Bundang-gu, Seongnam 13496, Korea.

E-mail: pshwang@chamc.co.kr

Copyright $\odot 2017$ The Korean Society for Aesthetic Plastic Surgery.

This is an Open Access article distributed under the terms of the Creative Commons Attribution Non-Commercial License (http://creativecommons.org/licenses/by-nc/4.0/) which permits unrestricted non-commercial use, distribution, and reproduction in any medium, provided the original work is properly cited. $\quad w w w . e-a a p s . o r g$ tinomyces. The disease is slow-spreading and induces granulomatous inflammation and the formation of multiple abscesses [2]. Actinomyces species are anaerobic, Gram-positive, filamentous bacteria. They usually inhabit the human oral cavity or respiratory and digestive tracts. Some conditions causing Actinomyces infection are trauma, oral surgery, tooth extraction, and poor dental hygiene. Because actinomycosis is not commonly encountered in the field of plastic surgery, it may be easily ignored. We report a case in which actinomycosis developed in a previously created facial dimple.

\section{CASE REPORT}

A 51-year-old woman presented with a palpable mass about $1 \mathrm{~cm}$ in size on her left cheek, which had gradually protruded into the buccal mucosal side over the preceding month. The patient had undergone facial dimple-creating surgery on both perioral areas at a local clinic 12 years previously. She had not experienced any complications postoperatively until she was diagnosed with rheumatoid 
arthritis (RA) and diabetes mellitus about 2 years previously. Since then, she took immunosuppressive disease-modifying antirheumatic drugs (DMARDs), including leflunomide and methotrexate. A physical examination demonstrated that the mass was located on the previously created dimple site (Fig. 1). Ultrasonography confirmed that the mass was a heterogeneously hypoechoic cystic lesion approximately $0.7 \times 0.7 \times 0.9 \mathrm{~cm}$ in size (Fig. 2 ). The mass was completely excised through an intraoral approach while the patient was under local anesthesia. The specimen was grossly described as a gray-yellow cystic mass containing non-absorbable suture material (\#4-0 nylon suture) (Fig. 3). There were no complications at the surgical site and the lesion did not recur during a 3-month follow-up period (Fig. 4). A histologic examination showed dark-stained foci, known as sulfur granules, which are characteristic of Actinomyces organisms. The filamentous nature of the Actinomyces organisms was noted in these sulfur granules (Fig. 5).

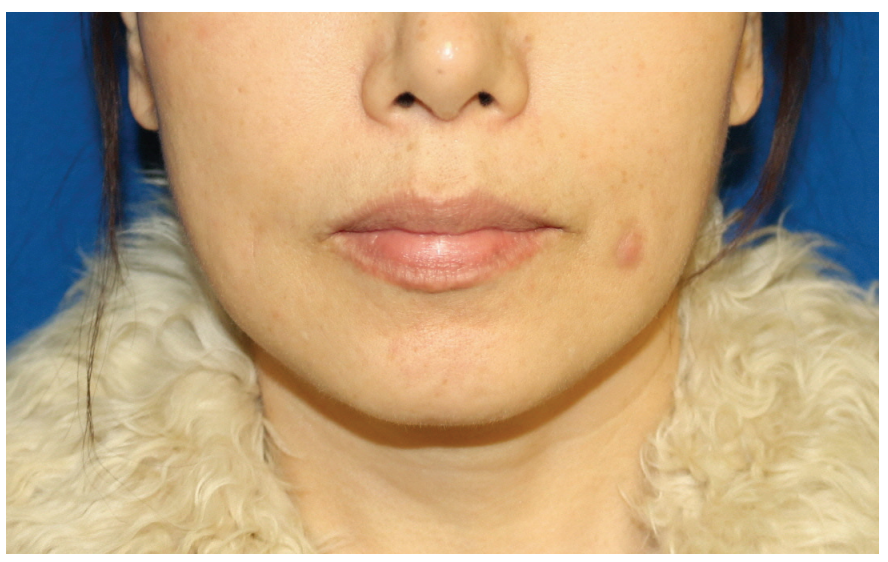

Fig. 1. Preoperative view showing the $1-\mathrm{cm}$ protruding mass on the created left dimple.

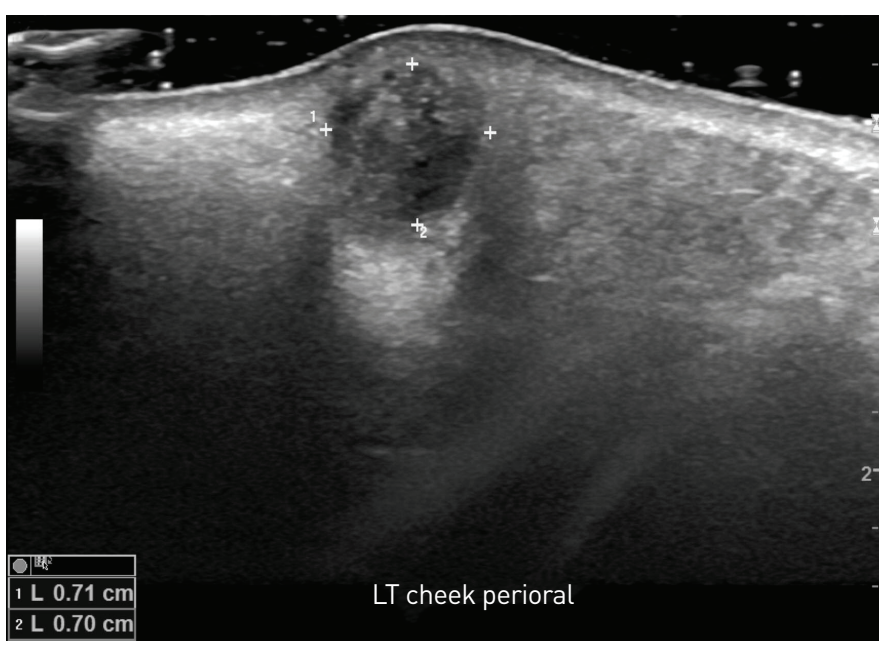

Fig. 2. Facial sonography showing an ill-defined, heterogeneously hypoechoic cystic lesion measuring $0.7 \times 0.7 \times 0.9 \mathrm{~cm}$.

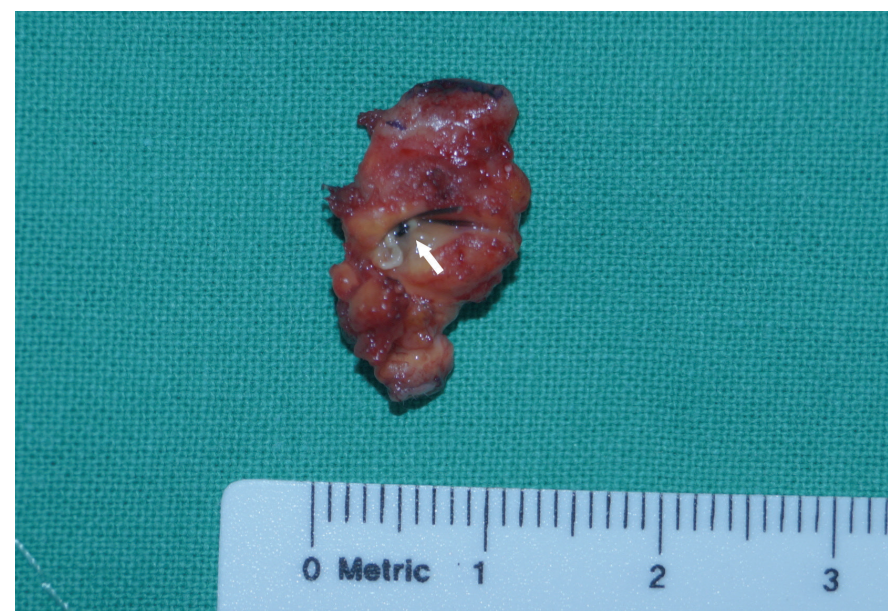

Fig. 3. A yellow, firm nodule with a nylon suture (white arrow) was observed on gross examination.

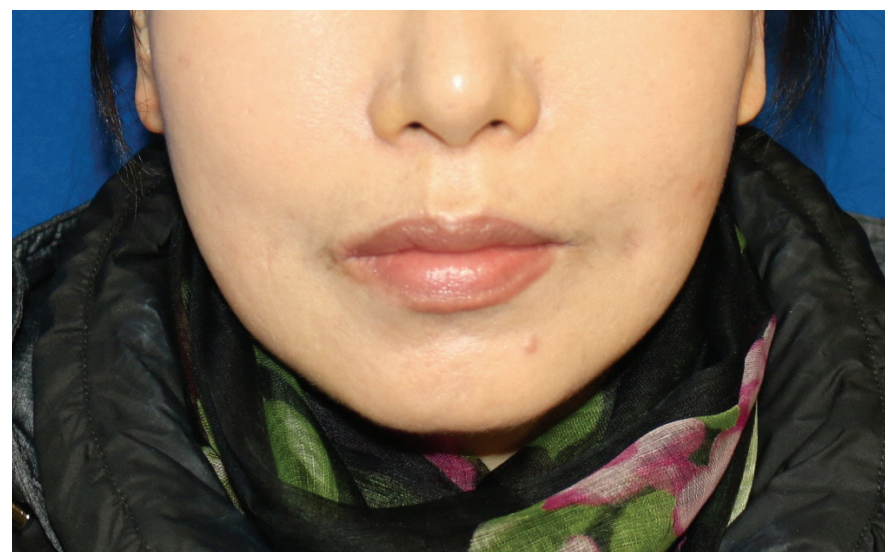

Fig. 4. Three-month postoperative view showing no recurrence of actinomycosis.

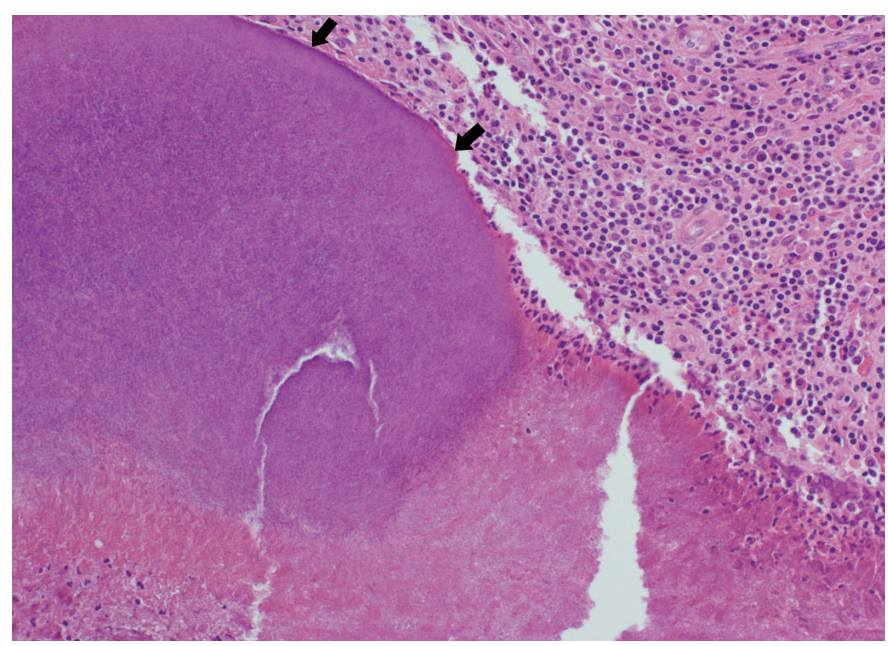

Fig. 5. The filamentous nature of the Actinomyces organisms in the sulfur granule (black arrows) was noted (hematoxylin and eosin stain $[H \& E], \times 200)$. 


\section{DISCUSSION}

Facial dimples are an attractive component of the face, and the demand for dimple creation surgery has gradually increased. Dimples are thought to be caused by the insertion of fascial bands of the zygomaticus major muscle into the dermis or by a dermal tethering effect of the zygomaticus major muscle, which may be bifid $[3,4]$. Dimples, however, may also be acquired or observed after focal trauma to the face. Since Boo-Chais report of a technique for creating facial dimples in 1962, several authors have described various techniques of creating perioral dimples. Bao et al. [5] reported a simple technique in which a syringe needle is used to connect the buccinator muscle to the dermis with non-absorbable suture materials. For long-term results, many surgeons prefer using non-absorbable suture materials, such as nylon or prolene. However, nonabsorbable suture material can increase the possibility of a foreign body granuloma or an inclusion cyst. In addition, excessive tension at the suture site may induce a mucosal defect due to mucosal ischemia. Finally, this condition provides an invasive route to submucosal or subcutaneous tissue [6].

In this case, the patient had not shown any complications following dimple creation surgery until she began taking immunosuppressive medicines for her rheumatoid arthritis. Because DMARDs target the immune system, they can weaken the immune system's ability to fight infections. Actinomycosis developed after the patient had been taking immunosuppressive medications for 2 years.

Actinomycosis is a chronic infectious disease that is characterized by anaerobic Gram-positive bacteria that inhabit the oral cavity. The microbe causes the infection by entering into the submucosal or deeper tissues through a mucosal defect caused by trauma, dental caries, or manipulation [7-9]. Although actinomycosis is not thought to be an opportunistic infection, it has most commonly been reported in immunocompromised patients, such as those with human immunodeficiency virus, leukemia, or RA [10,11]. Kolm et al. [12] reported a case of cervicofacial actinomycosis that occurred in an 86-year-old woman with RA undergoing immunosuppressive treatment with azathioprine and prednisone. Untreated, actinomycosis can induce excessive scarring, abscess formation, multiple skin sinuses, infiltration of adjacent tissue, and cervicofacial actinomycosis [6]. Some cases of oral actinomycosis following dimple creation surgery have been reported, but no reports have accurately presented its incidence worldwide. Saraf and Pillutia [13] reported the case of a patient who developed bilateral cheek abscesses 1 month following dimple creation surgery. Under general anesthesia, the cheek abscesses were drained by an intraoral approach, and the patient recovered in 3 weeks, but with a severe cheek scar.

When a mucosal actinomycotic sinus or route following dimple creation surgery is confirmed, early treatment with surgery and oral antibiotics is recommended. Recently, Kouketsu et al. [14] have recommended through irrigation with the antibiotic solution amox- icillin $(3 \times 0.5 \mathrm{~g} /$ day $)$ and long-term antibiotic therapy with amoxicillin for 2 months. It is important to maintain proper oral hygiene to prevent additional complications. Postoperative management should be performed strictly in patients with poor oral hygiene and dental caries, and especially in patients taking immunosuppressive medicine. Additionally, patients who have undergone surgical procedures with indwelling non-absorbable suture materials, especially sling operations using non-absorbable suture materials, are predisposed to actinomycosis. Surgeons should thus be aware of the possibility of actinomycosis when they encounter a mass that has developed in an area adjacent to non-absorbable suture materials.

\section{PATIENT CONSENT}

Patients provided written consent for the use of their images.

\section{REFERENCES}

1. Boo-Chai K. The facial dimple-clinical study and operative technique. Plast Reconstr Surg Transplant Bull 1962;30:281-8.

2. Nakagawa Y, Asada K, Doi Y, et al. Oral and maxillofacial actinomycosis: Clinical and bacteriological studies of 13 cases. Jpn J Oral Maxillofac Surg 1985;31:110-5.

3. Gassner HG, Rafii A, Young A, et al. Surgical anatomy of the face: implications for modern face-lift techniques. Arch Facial Plast Surg 2008; 10:9-19.

4. Pessa JE, Zadoo VP, Garza PA, et al. Double or bifid zygomaticus major muscle: anatomy, incidence, and clinical correlation. Clin Anat 1998;11:310-3.

5. Bao S, Zhou C, Li S, et al. A new simple technique for making facial dimples. Aesthetic Plast Surg 2007;31:380-3.

6. AbdullGaffar B, Ghandoor K, Ahli Q. Actinomycotic sinus of the buccal mucosa: a rare complication of cheek dimple creation. Aesthet Surg J 2014;34:Np80-2.

7. Alamillos-Granados FJ, Dean-Ferrer A, García-López A, et al. Actinomycotic ulcer of the oral mucosa: an unusual presentation of oral actinomycosis. Br J Oral Maxillofac Surg 2000;38:121-3.

8. Southwick GJ, Lister GD. Actinomycosis of the hand: a case report. J Hand Surg Am 1979;4:360-2.

9. Jain A, Narula V, Alam K, et al. Cervicofacial actinomycosis mimicking sebaceous cyst. BMJ Case Rep 2013;2013.

10. Oostman O, Smego RA. Cervicofacial actinomycosis: Diagnosis and management. Curr Infect Dis Rep 2005;7:170-4.

11. Acevedo F, Baudrand R, Letelier LM, et al. Actinomycosis: a great pretender. Case reports of unusual presentations and a review of the literature. Int J Infect Dis 2008;12:358-62.

12. Kolm I, Aceto L, Hombach M, et al. Cervicofacial actinomycosis: a long forgotten infectious complication of immunosuppression - report of a case and review of the literature. Dermatol Online J 2014;20:22640.

13. Saraf S, Pillutia R. Complication of dimple creation. Indian Dermatol 
Online J 2010;1:42-3.

14. Kouketsu A, Matsui A, Mori S, et al. Actinomycosis manifested in the buccal mucosa estimated with scraping cytology: A case report. J Oral Maxillofac Surg Med Pathol 2017;29:249-53. 